\title{
A SPECIAL CASE OF GENERALIZED HÖLDER FUNCTIONS
}

\author{
Maria Lupa \\ Institute of Mathematics, Czestochowa University of Technology \\ Częstochowa, Poland \\ maria.lupa@im.pcz.pl
}

\begin{abstract}
In this paper some properties of a special case of generalized Hölder functions, which belong to the space $W_{\gamma}[a, b]$, are considered. These functions are $r$-times differentiable and their $r$-th derivatives satisfy the generalized Hölder condition. The main result of the paper is a proof of the theorem that product of two functions belonging to the space $W_{\gamma}[a, b]$ also belongs to this space.
\end{abstract}

Keywords: Lipschitz condition, generalized Hölder condition, equivalency of norms

\section{Introduction}

In the paper we introduce a function space $W_{\gamma}[a, b]$ and consider and prove some of its properties.

In the articles [1-4] the authors discussed Nemytskii operator determined on various function spaces (cf. also $[5,6]$ ). For instance, it is shown there that in each function space $\operatorname{Lip}[a, b], C^{r}[a, b], B V[a, b]$ a generating function of this operator exists and is affine with respect to the second variable. A similar result is obtained in [7] for the $W_{\gamma}[a, b]$-space. These results are then applied in $[8,9]$ to prove the existence and uniqueness of the solution of a certain functional equation in $W_{\gamma}[a, b]$.

\section{The space $W_{\gamma}$ and its properties}

Let $[a, b]$ be a closed interval, where $a, b \in R, a<b, d:=b-a$. We assume that the following condition is fulfilled

$$
\begin{gathered}
(I) \gamma:[0, d] \rightarrow[0, \infty) \text { is increasing and concave, } \gamma(0)=0, \\
\lim _{t \rightarrow 0^{+}} \gamma(t)=\gamma(0), \lim _{t \rightarrow d^{-}} \gamma(t)=\gamma(d) .
\end{gathered}
$$

\section{Definition 1.}

Denote by $W_{\gamma}[a, b]$ the set of all $r$-times differentiable functions, where $r \in N$, defined on the interval $[a, b]$ with values in $R$, such that their $r$-th derivatives satisfy the following condition: there exists a constant $M \geq 0$ such that 


$$
\left|\varphi^{(r)}(x)-\varphi^{(r)}(\bar{x})\right| \leq M \gamma(|x-\bar{x}|), \bar{x}, x \in[a, b]
$$

where $\gamma$ fulfils condition $(\Gamma)$.

\section{Remark 1.}

It is easily seen that $W_{\gamma}[a, b]$ contains the class of all $r$-times differentiable functions $\varphi:[a, b] \rightarrow R$, whose $r$-th derivatives satisfy the Lipschitz condition on $[a, b]$. This class is denoted by $\operatorname{LipC}^{r}[a, b]$. Thus we have

$$
\operatorname{LipC}^{r}[a, b] \subset W_{\gamma}[a, b] \text {. }
$$

\section{Remark 2.}

Denote by $\gamma_{+}^{\prime}(0)$ the right derivative of $\gamma$ at $t=0$. By $(\Gamma)$ we have $0 \leq \gamma_{+}^{\prime}(0) \leq+\infty$.

For $\varphi \in W_{\gamma}[a, b]$ and by the condition $(\Gamma)$ we obtain

$$
\left|\varphi^{(r)}(x)-\varphi^{(r)}(\bar{x})\right| \leq M \gamma(|x-\bar{x}|) \leq M \gamma_{+}^{\prime}(0)|x-\bar{x}|, \bar{x}, x \in[a, b]
$$

i.e. $\varphi^{(r)}$ fulfils an ordinary Lipschitz condition with the constant $K=M \gamma_{+}^{\prime}(0)$.

Thus if $\varphi \in W_{\gamma}[a, b]$ and $\gamma_{+}^{\prime}(0)$ is finite then $\varphi \in \operatorname{Lip}^{r}[a, b]$. In view of Remark 1 we get $\operatorname{LipC}^{r}[a, b]=W_{\gamma}[a, b]$.

Therefore only the case $\gamma_{+}^{\prime}(0)=+\infty$ is of interest.

\section{Remark 3.}

The functions of the form $\gamma(t)=t^{\alpha}$, where $0<\alpha<1, t \in[0, d]$, fulfil the assumption $(\Gamma)$ and moreover $\gamma_{+}^{\prime}(0)=+\infty$. Therefore the condition (1) is called the generalized Hölder condition or the $\gamma-$ Hölder condition.

\section{Lemma 1.}

If $\varphi \in W_{\gamma}[a, b]$ and $\gamma_{+}^{\prime}(0)=+\infty$, then the functions $\varphi^{(k)}$, where $k=$ $=0,1, \ldots, r-1$, fulfil the generalized Hölder condition with a function $\gamma_{0}$ and the constants $L_{k}, k=0,1, \ldots, r-1$.

Proof.

By the condition $\gamma_{+}^{\prime}(0)=+\infty$, we have $\gamma(t) \geq t$ in a right neighbourhood of zero. Suppose that $\gamma(d) \geq d$. Then we have $\gamma(t) \geq t$ for $t \in[0, d]$.

Let $\gamma(d)<d$. Define the function

$$
\gamma_{1}(t):=\frac{d}{\gamma(d)} \gamma(t), t \in[0, d]
$$


It is easily seen that $\gamma_{1}$ fulfils the condition $(\Gamma)$ and $\gamma(t) \leq \gamma_{1}(t)$ for $t \in[0, d]$. Moreover, $\gamma_{1}(t) \geq t$ for $t \in[0, d]$. Clearly, if a function $\varphi$ fulfils the generalized Hölder condition with the same function $\gamma$ such that $\gamma(d)<d$ and with a constant $M$, then $\varphi$ fulfils the generalized Hölder condition with the function $\gamma_{1}$, defined by (2), and the same constant $M$.

Define

$$
\gamma_{0}= \begin{cases}\gamma & \gamma(d) \geq d \\ \gamma_{1} & \gamma(d)<d\end{cases}
$$

where $\gamma_{1}$ is defined by (2). Thus the functions $\varphi^{(k)}$, where $k=0,1, \ldots, r-1$, fulfil the generalized Hölder condition with the function $\gamma_{0}$ defined by (3), i.e. for $\bar{x}, x \in[a, b]$ we have

$$
\left|\varphi^{(k)}(x)-\varphi^{(k)}(\bar{x})\right| \leq L_{k}|x-\bar{x}| \leq L_{k} \gamma_{0}(|x-\bar{x}|), k=0,1, \ldots, r-1 .
$$

This completes the proof.

\section{Remark 4.}

The space $W_{\gamma}[a, b]$ with the norm

$$
\|\varphi\|:=\sum_{k=0}^{r}\left|\varphi^{(k)}(a)\right|+\sup \left\{\frac{\left|\varphi^{(r)}(x)-\varphi^{(r)}(\bar{x})\right|}{\gamma(|x-\bar{x}|)} ; x, \bar{x} \in[a, b], x \neq \bar{x}\right\}
$$

is a real normed vector space. Moreover, it is a Banach space $([5,6])$.

In the space $W_{\gamma}[a, b]$ we define the second norm by the formula

$$
\begin{gathered}
\|\varphi\|_{0}:=\sum_{k=0}^{r} \sup _{x \in[a, b]}\left|\varphi^{(k)}(x)\right|+ \\
+\sup \left\{\frac{\left|\varphi^{(r)}(x)-\varphi^{(r)}(\bar{x})\right|}{\gamma(|x-\bar{x}|)} ; x, \bar{x} \in[a, b], x \neq \bar{x}\right\}
\end{gathered}
$$

We will show the equivalence of norms (4) and (5).

\section{Proposition 1.} hold

The norms (4) and (5) are equivalent in $W_{\gamma}[a, b]$ and the following inequalities

$$
\|\varphi\| \leq\|\varphi\|_{0} \leq K_{1}\|\varphi\|
$$

where

$$
K_{1}=\max \left(\sum_{k=0}^{r+1} d^{k} ; \sum_{k=0}^{r+1}(\gamma(d))^{k}\right)
$$


Proof.

The inequality $\|\varphi\| \leq\|\varphi\|_{0}$ is obvious. For $\varphi \in W_{\gamma}[a, b]$ define the constants

$$
\begin{gathered}
M:=\sup \left\{\frac{\left|\varphi^{(r)}(x)-\varphi^{(r)}(\bar{x})\right|}{\gamma(|x-\bar{x}|)} ; x, \bar{x} \in[a, b], x \neq \bar{x}\right\}, \\
L_{k}:=\sup \left\{\frac{\left|\varphi^{(k)}(x)-\varphi^{(k)}(\bar{x})\right|}{|x-\bar{x}|} ; x, \bar{x} \epsilon[a, b], x \neq \bar{x}\right\}, k=0,1, \ldots, r-1 .
\end{gathered}
$$

Since for $\varphi \in W_{\gamma}[a, b]$ the following inequalities hold

$$
\begin{gathered}
\sup _{x \in[a, b]}\left|\varphi^{(r)}(x)\right| \leq\left|\varphi^{(r)}(a)\right|+M \gamma(d) \\
\sup _{x \in[a, b]}\left|\varphi^{(k)}(x)\right| \leq\left|\varphi^{(k)}(a)\right|+L_{k} d, k=0,1, \ldots, r-1,
\end{gathered}
$$

and by the mean value theorem we have

$$
L_{k} \leq \sup _{x \in[a, b]}\left|\varphi^{(k+1)}(x)\right|, \quad k=0,1, \ldots, r-1,
$$

thus we obtain

$$
\sup _{x \in[a, b]}\left|\varphi^{(k)}(x)\right| \leq\left|\varphi^{(k)}(a)\right|+\left|\varphi^{(k+1)}(a)\right| d+\cdots+\left|\varphi^{(r)}(a)\right| d^{r-k}+M d^{r-k} \gamma(d)
$$

In view of above inequalities we have

$$
\begin{gathered}
\|\varphi\|_{0} \leq \sum_{k=0}^{r} \sup _{x \in[a, b]}\left|\varphi^{(k)}(x)\right|+ \\
+M \sum_{i=0}^{r}\left|\varphi^{(i)}(a)\right| \sum_{k=0}^{i} d^{k}+M\left(\gamma(d) \sum_{k=0}^{r} d^{k}+1\right) .
\end{gathered}
$$

In the case $\gamma(d) \geq d$ we obtain

$$
\gamma(d) \sum_{k=0}^{r} d^{k}+1 \leq \sum_{k=0}^{r+1}(\gamma(d))^{k},
$$

then

$$
\|\varphi\|_{0} \leq \sum_{k=0}^{r+1}(\gamma(d))^{k}\|\varphi\| .
$$

In the case $\gamma(d)<d$ we have

$$
\gamma(d) \sum_{k=0}^{r} d^{k}+1 \leq \sum_{k=0}^{r+1} d^{k}
$$

therefore

$$
\|\varphi\|_{0} \leq \sum_{k=0}^{r+1} d^{k}\|\varphi\| .
$$

In view of (7) we obtain inequality (6). This completes the proof. 


\section{Proposition 2.}

If the function $f$ and $g$ belong to $W_{\gamma}[a, b]$, then their product $f \cdot g$ also belongs to this space and there exists a constant $K_{2}>0$ such that

$$
\|f \cdot g\|_{0} \leq K_{2}\|f\|_{0}
$$

where

$$
\begin{gathered}
K_{2}=\max \left(P_{r}, Q_{r}\right) \\
P_{r}=\max _{i=0, \ldots, r-1}\left(\|g\|_{0} ; \sup _{[a, b]}\left|g^{(i)}\right|+2 \sup _{[a, b]}\left|g^{(i+1)}\right|\right) \\
Q_{r}=\left(\frac{d}{\gamma(d)}\right)^{r-1} \max _{i=0, \ldots, r-1}\left(\left(\frac{d}{\gamma(d)}\right)^{r-1}\|g\|_{0} ; \sup _{[a, b]}\left|g^{(i)}\right|+2 \frac{d}{\gamma(d)} \sup _{[a, b]}\left|g^{(i+1)}\right|\right)
\end{gathered}
$$

Proof.

For $f, g \in W_{\gamma}[a, b]$ we have

$$
\begin{gathered}
\left|(f g)^{(r)}(x)-(f g)^{(r)}(\bar{x})\right|= \\
=\left|\sum_{i=0}^{r}\left(\begin{array}{l}
r \\
i
\end{array}\right) f^{(i)}(x) g^{(r-i)}(x)-\sum_{i=0}^{r}\left(\begin{array}{l}
r \\
i
\end{array}\right) f^{(i)}(\bar{x}) g^{(r-i)}(\bar{x})\right| \leq \\
\leq \sup _{x \in[a, b]}|f|\left|g^{(r)}(x)-g^{(r)}(\bar{x})\right|+ \\
+\sum_{i=1}^{r}\left(\begin{array}{l}
r \\
i
\end{array}\right) \sup _{x \in[a, b]}\left|f^{(i)}\right|\left|g^{(r-i)}(x)-g^{(r-i)}(\bar{x})\right|+ \\
+\sum_{i=0}^{r-1}\left(\begin{array}{c}
r \\
i
\end{array}\right) \sup _{x \in[a, b]}\left|g^{(r-i)}\right|\left|f^{(i)}(x)-f^{(i)}(\bar{x})\right|+\sup _{[a, b]}|g|\left|f^{(r)}(x)-f^{(r)}(\bar{x})\right|
\end{gathered}
$$

Let $L_{i}, K_{i}, i=0, \ldots, r-1$, denote Lipschitz constants of the functions $f^{(i)}$ and $g^{(i)}$ respectively, and by $M_{f}, M_{g}$ denote $\gamma$-Hölder constants of the functions $f^{(r)}$ and $g^{(r)}$ respectively. Put

$$
\begin{aligned}
& F_{i}:=\sup _{[a, b]}\left|f^{(i)}\right|, i=0, \ldots, r, \\
& G_{i}:=\sup _{[a, b]}\left|g^{(i)}\right|, i=0, \ldots, r .
\end{aligned}
$$

Thus by Lemma 1 we get

$$
\begin{aligned}
\mid(f g)^{(r)}(x)- & (f g)^{(r)}(\bar{x}) \mid \leq F_{0} M_{g} \gamma(|x-\bar{x}|)+\sum_{i=1}^{r}\left(\begin{array}{l}
r \\
i
\end{array}\right) F_{i} K_{r-i} \gamma_{0}(|x-\bar{x}|)+ \\
& +\sum_{i=0}^{r-1}\left(\begin{array}{c}
r \\
i
\end{array}\right) G_{r-i} L_{i} \gamma_{0}(|x-\bar{x}|)+G_{0} M_{f} \gamma(|x-\bar{x}|),
\end{aligned}
$$

and by definition (3) of function $\gamma_{0}$ there is $f \cdot g \in W_{\gamma}[a, b]$. 
Using mathematical induction we prove the inequality (8).

For $r=1$ we have

$$
\begin{aligned}
& \|f \cdot g\|_{0} \leq \sup _{[a, b]}|f| \sup _{[a, b]}|g|+\sup _{[a, b]}|f| \sup _{[a, b]}\left|g^{\prime}\right|+\sup _{[a, b]}\left|f^{\prime}\right| \sup _{[a, b]}|g|+ \\
& +\sup \frac{\left|f^{\prime}(x) g(x)+f(x) g^{\prime}(x)-f^{\prime}(\bar{x}) g(\bar{x})-f(\bar{x}) g^{\prime}(\bar{x})\right|}{\gamma(|x-\bar{x}|)} \leq \\
& \quad \leq \sup _{[a, b]}|f|\left(\sup _{[a, b]}|g|+\sup _{[a, b]}\left|g^{\prime}\right|\right)+\sup _{[a, b]}\left|f^{\prime}\right| \sup _{[a, b]}|g|+ \\
& +\sup _{[a, b]}|f| \sup \frac{\left|g^{\prime}(x)-g^{\prime}(\bar{x})\right|}{\gamma(|x-\bar{x}|)}+\sup _{[a, b]}|g| \sup \frac{\left|f^{\prime}(x)-f^{\prime}(\bar{x})\right|}{\gamma(|x-\bar{x}|)}+ \\
& +\sup _{[a, b]}\left|f^{\prime}\right| \sup \frac{|g(x)-g(\bar{x})|}{\gamma(|x-\bar{x}|)}+\sup _{[a, b]}\left|g^{\prime}\right| \sup \frac{|f(x)-f(\bar{x})|}{\gamma(|x-\bar{x}|)}
\end{aligned}
$$

where supremes of the above rational expressions are for $x, \bar{x} \in[a, b], x \neq \bar{x}$. (In the next inequalities in this proof the same notations are used).

If $\gamma(d) \geq d$ then the following inequalities hold the function $g$ :

$$
\sup \frac{|g(x)-g(\bar{x})|}{\gamma(|x-\bar{x}|)} \leq \sup \frac{|g(x)-g(\bar{x})|}{|x-\bar{x}|} \leq \sup _{[a, b]}\left|g^{\prime}\right|
$$

and the same for function $f$. From the above we obtain

$$
\begin{aligned}
& \|f \cdot g\|_{0} \leq \sup _{[a, b]}|f|\left(\left(\sup _{[a, b]}|g|+\sup _{[a, b]}\left|g^{\prime}\right|+\sup \frac{\left|g^{\prime}(x)-g^{\prime}(\bar{x})\right|}{\gamma(|x-\bar{x}|)}\right)+\right. \\
& +\sup _{[a, b]}\left|f^{\prime}\right|\left(\sup _{[a, b]}|g|+2 \sup _{[a, b]}\left|g^{\prime}\right|\right)+\sup \frac{\left|f^{\prime}(x)-f^{\prime}(\bar{x})\right|}{\gamma(|x-\bar{x}|)} \sup _{[a, b]}|g| \leq \\
& \quad \leq\|f\|_{0} \max \left(\|g\|_{0} ; \sup _{[a, b]}|g|+2 \sup _{[a, b]}\left|g^{\prime}\right|\right)=P_{1}\|f\|_{0}
\end{aligned}
$$

If $\gamma(d)<d$, by (3) we have the following inequalities

$$
\sup \frac{|g(x)-g(\bar{x})|}{\gamma_{0}(|x-\bar{x}|)} \leq \sup \frac{|g(x)-g(\bar{x})|}{|x-\bar{x}|} \leq \sup _{[a, b]}\left|g^{\prime}\right|
$$

and using definition (2) we obtain

$$
\sup \frac{|g(x)-g(\bar{x})|}{\gamma(|x-\bar{x}|)} \leq \frac{d}{\gamma(d)} \sup _{[a, b]}\left|g^{\prime}\right|
$$

(similarly for the function $f$ ). 
Therefore we have

$$
\begin{gathered}
\|f \cdot g\|_{0} \leq \sup _{[a, b]}|f|\left(\leq \sup _{[a, b]}|f|\left(\sup _{[a, b]}|g|+\sup _{[a, b]}\left|g^{\prime}\right|+\sup \frac{\left|g^{\prime}(x)-g^{\prime}(\bar{x})\right|}{\gamma(|x-\bar{x}|)}\right)+\right. \\
\left.+\sup _{[a, b]}\left|f^{\prime}\right| \sup _{[a, b]}|g|+2 \frac{d}{\gamma(d)} \sup _{[a, b]}\left|g^{\prime}\right|\right)+\sup \frac{\left|f^{\prime}(x)-f^{\prime}(\bar{x})\right|}{\gamma(|x-\bar{x}|)} \sup _{[a, b]}|g| \leq \\
\quad \leq\|f\|_{0} \max \left(\|g\|_{0} ; \sup _{[a, b]}|g|+2 \frac{d}{\gamma(d)} \sup _{[a, b]}\left|g^{\prime}\right|\right)=Q_{1}\|f\|_{0}
\end{gathered}
$$

Putting

$$
K_{2}=\max \left(P_{1}, Q_{1}\right)
$$

we get the inequality (8) for $r=1$.

Let functions $f, g$ have $(k+1)$-order derivatives. Denote by $\|\cdot\|_{0, k}$ the norm $\|\cdot\|_{0}$ for the functions $k$ times differentiable. Suppose that the inequality (8) is true for $k \geq 1, k \in N$, i.e.

$$
\|f \cdot g\|_{0, k} \leq K_{2}\|f\|_{0, k}
$$

where

$$
\begin{gathered}
K_{2}=\max \left(P_{k}, Q_{k}\right) \\
P_{k}=\max _{i=0, \ldots, k-1}\left(\|g\|_{0, k} ; \sup _{[a, b]}\left|g^{(i)}\right|+2 \sup _{[a, b]}\left|g^{(i+1)}\right|\right) \\
Q_{k}=\left(\frac{d}{\gamma(d)}\right)^{k-1} \max _{i=0, \ldots, k-1}\left(\left(\frac{d}{\gamma(d)}\right)^{k-1}\|g\|_{0, k} ; \sup _{[a, b]}\left|g^{(i)}\right|+2 \frac{d}{\gamma(d)} \sup _{[a, b]}\left|g^{(i+1)}\right|\right)
\end{gathered}
$$

We prove that the inequality $(8)$ holds for $(k+1)$. We have

$$
\|f \cdot g\|_{0, k+1} \leq \sup _{[a, b]}|f| \sup _{[a, b]}|g|+\left\|f \cdot g^{\prime}\right\|_{0, k}+\left\|f^{\prime} \cdot g\right\|_{0, k}
$$

If $\gamma(d) \geq d$ then the following inequality holds

$$
\begin{gathered}
\|f \cdot g\|_{0, k+1} \leq \sup _{[a, b]}|f| \sup _{[a, b]}|g|+ \\
+\|f\|_{0, k} \max _{i=0, . ., k-1}\left(\left\|g^{\prime}\right\|_{0, k} ; \sup _{[a, b]}\left|g^{(i+1)}\right|+2 \sup _{[a, b]}\left|g^{(i+2)}\right|\right)+ \\
+\left\|f^{\prime}\right\|_{0, k} \max _{i=0, \ldots, k-1}\left(\|g\|_{0, k} ; \sup _{[a, b]}\left|g^{(i)}\right|+2 \sup _{[a, b]}\left|g^{(i+1)}\right|\right)
\end{gathered}
$$


Since

$$
\|f\|_{0, k} \leq\|f\|_{0, k+1} ;\left\|f^{\prime}\right\|_{0, k} \leq\left\|f^{\prime}\right\|_{0, k+1}
$$

(the same inequalities hold for the function $g$ ) thus we have

$$
\begin{gathered}
\|f \cdot g\|_{0, k+1} \leq\|f\|_{0, k+1} \max _{i=0, \ldots, k}\left(\|g\|_{0, k+1} ; \sup _{[a, b]}\left|g^{(i)}\right|+2 \sup _{[a, b]}\left|g^{(i+1)}\right|\right)= \\
=P_{k+1}\|f\|_{0, k+1}
\end{gathered}
$$

In the case $\gamma(d)<d$ we have

$$
\|f\|_{0, k} \leq \frac{d}{\gamma(d)}\|f\|_{0, k+1} ;\left\|f^{\prime}\right\|_{0, k} \leq\left\|f^{\prime}\right\|_{0, k+1}
$$

(the same inequalities hold for the function $g$ ). Therefore

$$
\begin{gathered}
\|f \cdot g\|_{0, k+1} \leq \sup _{[a, b]}|f| \sup _{[a, b]}|g|+ \\
+\|f\|_{0, k}\left(\frac{d}{\gamma(d)}\right)^{k} \max _{i=0, \ldots, k-1}\left(\left(\frac{d}{\gamma(d)}\right)^{k-1}\left\|g^{\prime}\right\|_{0, k} ; \sup _{[a, b]}\left|g^{(i+1)}\right|\right. \\
\left.+2 \frac{d}{\gamma(d)} \sup _{[a, b]}\left|g^{(i+2)}\right|\right)+ \\
+\left\|f^{\prime}\right\|_{0, k}\left(\frac{d}{\gamma(d)}\right)^{k-1} \max _{i=0, \ldots, k-1}\left(\left(\frac{d}{\gamma(d)}\right)^{k-1}\|g\|_{0, k} ; \sup _{[a, b]}\left|g^{(i)}\right|\right. \\
\left.+2 \frac{d}{\gamma(d)} \sup _{[a, b]}\left|g^{(i+1)}\right|\right) \leq \\
\leq\|f\|_{0, k+1}\left(\frac{d}{\gamma(d)}\right)^{k} \max _{i=0, \ldots, k-1}\left(\left(\frac{d}{\gamma(d)}\right)^{k}\|g\|_{0, k+1} ; \sup _{[a, b]}\left|g^{(i)}\right|+\right. \\
\left.+2 \frac{d}{\gamma(d)} \sup _{[a, b]}\left|g^{(i+1)}\right|\right)=Q_{k+1}\|f\|_{0, k+1}
\end{gathered}
$$

Putting $K_{2}=\max \left(P_{k+1}, Q_{k+1}\right)$ we get the inequality $(8)$ for $(k+1)$. This completes the proof.

\section{Conclusions}

In this paper some properties of the Banach space $W_{\gamma}[a, b]$ have been presented. They will be applied in the forthcoming papers. 


\section{References}

[1] Matkowska A., On characterization of Lipschitzian operator of substitution in the class of Hölders functions, Scientific of Łódź Technical University 1984, 17, 81-85.

[2] Matkowski J., Functional equations and Nemytskii operators, Funkcialaj Ekvacioj 1982, 25, 127-132.

[3] Matkowski J., Form of Lipschitz operators of substitution in Banach space of differentiable functions, Zeszyty Naukowe Politechniki Łódzkiej, Matematyka, 1982, 25, 127-132.

[4] Matkowski J., Miś J., On a characterization of Lipschitzian operators of substitution in the space BV[a,b], Math. Nachr. 1984, 117, 155-159.

[5] Appell J., Banaś J., Merentes N., Bounded Variation and Around, De Gruyter Studies in Nonlinear Analysis and Applications, Volume 17, Würzburg 2014.

[6] Appell J., Zabrejko P.P., Nonlinear Superposition Operators, Cambridge University Press, Cambridge-New York-Port Chester-Melbourne-Sydney 1990.

[7] Lupa M., Form of Lipschitzian operator of substitution in some class of functions, Zeszyty Naukowe Politechniki Łódzkiej, Matematyka, 1990, 21, 87-96.

[8] Lupa M., On solutions of a functional equation in a special class of functions, Demonstratio Mathematica 1993, XXVI, 1, 137-147.

[9] Lupa M., $\mathrm{W}_{\gamma}$ - solutions of linear Iterative Functional Equations, Demonstratio Mathematica 1994, XXVII, 2, 417-425. 